\title{
Forest bathing in cardiovascular diseases - a narrative review
}

\section{DOGARU Gabriela}

1."Iuliu Hatieganu" University of Medicine and Pharmacy, Cluj-Napoca, Romania 2.Clinical Rehabilitation Hospital, Cluj-Napoca, Romania

Corresponding author: DOGARU Gabriela, E-mail: $\underline{\text { dogarugabrielaumf@gmail.com }}$

(cc) BY-NC-ND Balneo Research Journal DOI: http://dx.doi.org/10.12680/balne0.2020.356 Vol.11, No.3, September 2020 p: 299-303

Peer reviewer: Olga SURDU, Romanian Association of Balneology, office@bioclima.ro

\begin{abstract}
Introduction.People of all ages have always enjoyed the forest, due to the peaceful environment, mild climate, extremely beautiful landscapes, fresh air, pleasant flower and plant scents. Over the past years, a new medical science termed forest medicine has developed, as an interdisciplinary science belonging to the fields of alternative medicine, environmental medicine and preventive medicine, which comprises the effects of forest environments on human health. However, beyond its use for pleasure, there is increasing evidence suggesting that the forest can offer very many benefits for health, including in cardiovascular diseases. Objectives. To bring to attention a less known and studied therapeutic issue, i.e. the effects of forest bathing on the human body, especially in cardiovascular diseases. Material and method. Given the heterogeneous nature of the evidence in the literature, these findings should be put together in order to allow an adequate interpretation. Thus, we reviewed the observational clinical studies (on prospective cohorts, case-control studies, retrospective cohorts), the randomized controlled trials (RCT) and nonrandomized controlled trials in PubMed, over the past 20 years, until June 2020, focusing on the effects of the forest in cardiovascular diseases. The search terms included forest, forest bathing, cardiovascular effects, blood pressure, diabetes mellitus, coronary artery disease, stress, psychological effects. Results and discussions. The results of the studies show that the benefits of the forest on the cardiovascular system are obvious regardless of age, sex, socioeconomic environment or previous exposure to a natural environment. Also, walking in the forest environment can promote cardiovascular relaxation by facilitating the parasympathetic nervous system and by suppressing the sympathetic nervous system. In addition, forest therapy can be effective in reducing negative psychological symptoms. The current literature supports the benefits of exposure to nature and green environments for human health, through the effects on: the immune system (increase of natural killer cells/cancer prevention); the cardiovascular system (hypertension/coronary artery disease); the respiratory system (allergies and respiratory diseases); depression and anxiety (mood disorders and stress); mental relaxation. Conclusions. The studies conducted show the cardiovascular benefits associated with continuous immersion in nature, but further research regarding this aspect is definitely required.
\end{abstract}

Key words: forest, forest bathing, cardiovascular effects, blood pressure, forest walking,

\section{Introduction}

People of all ages have always enjoyed the forest, due to the peaceful environment, mild climate, extremely beautiful landscapes, fresh air, pleasant flower and plant scents. In Japan, starting with 2004, a lot of studies have been conducted to investigate the effects of forest environments on human health. Over the past years, a new medical science termed forest medicine has developed, as an interdisciplinary science belonging to the fields of alternative medicine, environmental medicine and preventive medicine, which comprises the effects of forest environments on human health (1).

In Germany, for the first time in Europe, healing forests have been developed and cultivated in the Mecklenburg-Vorpommern area. Also, in
Heringsdorf on the Baltic Sea coast, Germany has already created a first spa resort with forest bathing indications. Studies conducted in transcontinental Japan and China have evidenced a multitude of physiological and psychological benefits associated with the practice of Shinrin-Yoku (SY), known as forest bathing. SY is a traditional Japanese practice of immersion in nature through all five senses. In the 1980's, SY emerged in Japan as an essential component of preventive and curative medicine (1). The popularization of forest bathing in Japan through easy access to the forest environment and governmental recommendations resulted in a reduction of acute psychological disorders due to the time spent in green spaces (1). 
Objectives. To bring to attention a less known and studied therapeutic issue, i.e. the effects of forest bathing on the human body, especially in cardiovascular diseases.

\section{Material and method.}

However, beyond its use for pleasure, there is increasing evidence suggesting that the forest can offer very many benefits for health. Over the past decade, a considerable number of findings from various countries regarding the potential benefits of the forest on health, as well as the possible biological mechanisms underlying these effects have been reported. Given the heterogeneous nature of the evidence in the literature, these findings should be put together in order to allow an adequate interpretation. Thus, we reviewed the observational clinical studies (on prospective cohorts, case-control studies, retrospective cohorts), the randomized controlled trials (RCT) and non-randomized controlled trials

Search strategy. For our research, we used the PubMed database. We selected and evaluated all the articles resulting from a systematic search. The search was aimed at finding articles published over the past 20 years, from 2000 to 2020 , using a certain key word combination. The search terms included forest, forest bathing, cardiovascular effects, blood pressure, diabetes mellitus, coronary artery disease, stress, psychological effects.

Selection criteria. We used several inclusion criteria: a) articles written in English; b) studies of the effects of forest bathing on cardiovascular diseases; c) studies in which the forest was used as a complementary therapy. We also used exclusion criteria: a) articles reporting no data; b) studies conducted on animals; c) effects of the forest on stress and psychological effects.

Data extraction. A screening of the titles found using the search strategy, without any difference in their findings, was performed. The articles relevant for the subject of the present study were selected using the inclusion and exclusion criteria. A total number of 21 articles were found. After the application of both inclusion and exclusion criteria, 8 articles were excluded because they were not relevant for our subject, presenting only effects on stress and psychological effects. The other 13 articles were analyzed and after being read, they were all taken into consideration for the current study. Some studies showed the use of forest bathing in certain cardiovascular diseases.

\section{Results and discussions}

Forest bathing can be considered a form of nature therapy. According to the model of Song, Ikei and Miyazaki (2), the concept of nature therapy clearly defines "a set of practices aimed at achieving "preventive medical effects" through exposure to natural stimuli that confer a state of physiological relaxation and improve the weakened immune functions to prevent diseases". The conceptual model of nature therapy starts with a "state of stress" and then highlights the "restorative effects" of nature (forests, flowers, etc.) in which an improvement of "physiological relaxation" and "recovery of immune function" (with individual differences) is hypothesized. These responses to nature are subsequently incorporated in the model of evidencebased medicine (EBM), leading to the "preventive medical effect" (2).

Studies over the past 10 years regarding the effects of the forest on the cardiovascular system and the autonomic nervous system (ANS) started with basic cardiac monitoring, then involved the correlation of the monitored cardiac parameters with the parasympathetic nervous system (PNS), the sympathetic nervous system (SNS), and subsequently, more extensive studies on the effects of SY on specific pathological conditions: arterial hypertension (HTN), coronary artery disease (CAD), and chronic obstructive pulmonary disease (COPD) (1).

The aim of a study (3) was to examine the physiological effects of forests on autonomic nervous activity. Twelve male students $(21.8 \pm 0.8$ years) were included in the study; six subjects were sent to a forest area and the other six, to an urban area. The heart rate variability (HRV), blood pressure and pulse rate were measured as physiological indices. The results showed that the pulse rate and diastolic blood pressure were significantly lower in subjects in the forest area compared to those in the urban area, the forest allowing effective relaxation of the humans (3). According to another study (4), 625 Japanese men from 57 forest locations and 57 urban locations in Japan had an $80 \%$ increase in the parasympathetic indicators of HRV in the case of forest locations, demonstrating in this way that the forest was more effective in reducing stress indicators than the urban environment. Blood pressure and PR also decreased in the forest environment compared to the urban environment (4). 
It is considered that the evolution of information technology causes great stress to modern people, and controlling this stress can become an important problem. The aim of a study was to examine the psychological and physiological benefits of the interaction with indoor plants. The study subjects were 24 young male adults aged $24.9 \pm 2.1$ years. The first group (12 subjects) performed indoor plant gardening tasks, while the second group (12 subjects) performed a computer task. Psychological evaluation was carried out using the semantic differential method (SDM), and physiological evaluation was based on heart rate variability and blood pressure. The results of the study suggested that active interaction with indoor plants can reduce physiological and psychological stress compared to mental work. This occurs by suppression of the sympathetic nervous system activity and diastolic blood pressure, and promotion of comfortable, calm and natural feelings (5).

The study of Tsunetsugu et al. (6), conducted in 12 male students aged between 21 and 23 years (mean +/- SD: $22.0+/-1.0$ years), investigated the physiological effects of "Shinrin-yoku" by assessing blood pressure, pulse, heart rate variability (HRV), salivary cortisol concentration and salivary immunoglobulin A. Subjective feelings of being "comfortable", "calm" and "refreshed" were also evaluated by a questionnaire. The results showed that in the forest area compared to the urban area, blood pressure and pulse rate were significantly lower, and salivary cortisol concentration was significantly decreased. These physiological responses suggest that sympathetic nervous system activity was suppressed and that parasympathetic nervous system activity was improved in the forest area, and also, that "Shinrin-yoku" reduced the stress level. Based on subjective evaluation, the "comfortable", "calm" and "refreshing" feelings were significantly greater in the forest area (6). Also, a study on 17 women aged over 40 years conducted by Ochiai et al. (7) found a global reduction of heart rate (HR) after a one-day forest therapy program. These studies demonstrate cardiovascular benefits for both sexes. Kardan et al. (8) performed a correlation analysis of the data acquired by the Canadian Ontario Health Study. The high-resolution satellite images showed that residents in areas with a higher tree density suffer from fewer cardiometabolic diseases than residents in areas with fewer trees (8). The benefits of SY on the cardiovascular system are obvious regardless of age, sex, socioeconomic environment or previous exposure to a natural environment (1).

A significant number of studies have evidenced the effects of SY and nature therapy (NY) on specific pathological conditions, including HTN, CAD, COPD and type 2 diabetes mellitus (DMII). The study carried out by Mao et al. (9) aimed to provide scientific evidence supporting the efficacy of forest bathing as a natural therapy for arterial hypertension. Thus, 24 elderly patients diagnosed with essential hypertension were randomized to two groups of 12 patients. A group was sent to a forest to experience a 7 day/7 night trip, and the other group was sent to a city in the Hangzhou area for control. Blood pressure indicators, pathological factors related to cardiovascular diseases, including endothelin-1, homocysteine, renin, angiotensinogen, angiotensin II, angiotensin II type 1 receptor, angiotensin II type 2 receptor, as well as the inflammatory cytokines interleukin- 6 and tumor necrosis $\alpha$ were measured. The results of the study showed that subjects exposed to the forest environment had a significant reduction of blood pressure compared to the urban group; the bioindicator values of the subjects exposed to the forest environment were also lower than those of the subjects in the urban control group and their baseline levels (9).

In order to test the hypothesis that walking in a park has a greater positive effect on the hemodynamic parameters of patients with coronary artery disease (CAD) than walking in an urban environment, a study on 20 stable patients with CAD was conducted. These were randomized to two groups: 30 minutes of walking for 7 consecutive days in a city park or on a busy street. The Wilcoxon signed test was used to study the short-term changes (30 $\mathrm{min}$ ) and cumulative changes (after 7 consecutive days of exposure) in hemodynamic parameters at rest in different environments. It was concluded that walking in a park had a greater positive effect on the cardiac function of patients with CAD than walking in an urban environment, which suggests that cardiac rehabilitation by walking in green areas following coronary events should be encouraged (10).

Another study (11) determined the effects of forest bathing on elderly patients with chronic obstructive pulmonary disease (COPD), through a reduction of inflammation and stress hormones. The patients were randomized to two groups. One group was sent to the forest and the other one was sent to a control urban area. 
Flow cytometry and stress hormones were evaluated. In the forest group, a significant decrease in perforin and granzyme B expression was found, accompanied by a reduction of proinflammatory cytokines and stress hormones.

In a longitudinal study including 48 adults diagnosed with DMII, it was found that blood glucose values decreased after multiple SY sessions, indicating a significant correlation between $\mathrm{SY}$ and the reduction of blood glucose levels $(1,12)$. In another study, 48 participants, 16 men and 32 women, with a mean age of 66.8 years, diagnosed with DMII, walked 3 to $6 \mathrm{~km}$ nine times a week over a period of 6 years. There were no statistically significant differences in the glucose levels or the HbAlc levels of the subjects between the shorter and longer walks. However, the mean blood glucose levels in both groups before and after the forest walks decreased by $79 \pm 10 \mathrm{mg} / \mathrm{dL}$ and $76 \pm 7 \mathrm{mg} / \mathrm{dL}$ respectively $(1,13)$.

Despite the increasing attention paid to forest therapy as an alternative therapy, there is very little evidence of its therapeutic effects. Consequently, a study focused on clarifying the health benefits of forest walking regarding cardiovascular reactivity. Forty-eight young adult men participated in the study for two days. Heart rate variability (HRV) was measured to investigate physiological reactivity to walking as an indicator of autonomic nervous activity. Based on the frequency analysis of the RR interval, high frequency (HF) data were used as an indicator of parasympathetic nervous activity, and the low frequency/high frequency (LF/HF) ratio was used as an indicator of sympathetic nervous activity. Heart rate was also investigated using R-R interval data. HRV and cardiac rhythm were continuously recorded during walking. Statistical analysis showed the fact that forest walking significantly increased $\ln$ (HF) values and significantly decreased $\ln (\mathrm{LF} / \mathrm{HF})$ values compared to urban walking. Heart rate during forest walking was significantly lower than in the control group. The conclusion of the study was that forest walking can promote cardiovascular relaxation by facilitating the parasympathetic nervous system and suppressing the sympathetic nervous system. In addition, forest therapy can be effective in reducing negative psychological symptoms (1).

The current literature supports the benefits of exposure to nature and green environments for human health, through the effects on: the immune system (increase of natural killer cells/cancer prevention); the cardiovascular system (hypertension/coronary artery disease); the respiratory system (allergies and respiratory diseases); depression and anxiety (mood disorders and stress); mental relaxation. Nature therapy as a health promotion method and a universal health model is involved in reducing the "state of stress" and "technostress" of our days (1). Western researchers are invited to conduct empirical studies regarding the therapeutic benefits associated with forest bathing and health care service providers/students are encouraged to take into consideration its practice to reduce stress and potential disconnection (1).

In Romanian spa resorts, climate therapy is indicated in the form of: heliotherapy by exposure of the body to solar radiation (infrared and ultraviolet radiation) for prophylactic and/or therapeutic purposes; aerotherapy by partial or total outdoor exposure without solar radiation (in the shade or during hours/days without sun); walking, which is a form of movement therapy, associated with aerotherapy. For walking, standardized routes are established regarding the length, the level difference, the slopes included, the walking pace, depending on the disease. The walking routes are marked, placed in forest areas with attractive landscape elements $(14,15,16)$. The natural therapeutic factors consisting of carbonated mineral water baths, mofettes, climate therapy, through forest walking along with medical physical culture, indicated in the rehabilitation of post-stroke patients, had a beneficial effect on clinical and functional symptoms, improving the quality of walking and balance, functionality in a patient who suffered a stroke five years before and was followed up for three years, during participation in an annual medical rehabilitation program. The rehabilitation treatment including walking improves quality of life in post-stroke survivors $(16,17)$, as well as in patients with chronic obliterative arteriopathy (18).

\section{Conclusions}

The studies conducted show the benefits associated with continuous immersion in nature on some cardiovascular diseases, but this aspect definitely requires further research. Global studies are needed in order to provide new evidence of the relationship between the forest - forest bathing - and clinical therapeutic effects. 


\section{References}

1. Margaret M. Hansen, Reo Jones and Kirsten Tocchini. Shinrin-Yoku (Forest Bathing) and Nature Therapy: A State-of-the-Art Review. Int J Environ Res Public Health. 2017 Aug; 14(8): 851. doi: 10.3390/ijerph14080851.

2. Song, C.; Ikei, H., Miyazaki, Y. Physiological Effects of Nature Therapy: A Review of the Research in Japan. Int. J. Environ. Res. Public Health; 2016 Aug 3;13(8):781. doi: 10.3390/ijerph13080781.

3. Park, B.- J., Tsunetsugu, Y.; Kasetani, T., Morikawa, T., Kagawa, T., Miyazaki, Y. Physiological effects of forest recreation in a young conifer forest in Hinokage Town, Japan. Silva Fenn. 2009; 43: 291-301.

4. Kobayashi H., Song C., Ikei H., Kagawa T., Miyazaki Y. Analysis of Individual Variations in Autonomic Responses to Urban and Forest Environments. Evid. Based Complement. Altern. Med. 2015:671094. doi: 10.1155/2015/671094.

5. Lee, M., Lee, J., Park, B., Miyazaki, Y. Interaction with indoor plants may reduce psychological and physiological stress by suppressing autonomic nervous system activity in young adults: A randomized crossover study. J. Physiol. Anthropol. 2015 Apr 28;34(1):21. doi: 10.1186/s40101-015-0060-8.

6. Tsunetsugu, Y., Park, B., Ishii, H., Hirano, H., Kagawa, T., Miyazaki, Y. Physiological effects of Shinrin-yoku (taking in the atmosphere of the forest) in an old-growth broadleaf forest in Yamagata Prefecture, Japan. J. Physiol. Anthropol. 2007;26: 135142.

7. Ochiai, H., Ikei, H., Song, C., Kobayashi, M., Miura, T., Kagawa, T., Li, Q., Kumeda, S., Imai, M., Miyazaki, Y. Physiological and Psychological Effects of a Forest Therapy Program on Middle-Aged Females. Int. J. Environ. Res. Public Health .2015; 12:1522215232.

8. Kardan, O., Gozdyra, P., Misic, B., Moola, F., Palmer, L.J., Paus, T., Berman, M.G. Neighborhood greenspace and health in a large urban center. Sci. Rep..2015;5:1-14.

9. Mao, G., Cao, Y., Lan, X., He, Z., Chen, Z., Wang, Y., Hu, X., Lv, Y., Wang, G., Yan, J. Therapeutic effect of forest bathing on human hypertension in the elderly. J. Cardiol. 2012; 60:495-502.
10. Grazuleviciene, R., Vencloviene, J., Kubilius, R., Grizas, V., Dedele, A., Grazulevicius, T., Ceponiene, I., Tamuleviciute-Prasciene, E., Nieuwenhuijsen, M.J., Jones, M., et al. The Effect of Park and Urban Environments on Coronary Artery Disease Patients: A Randomized Trial. BioMed Res. Int. 2015;2015:403012. doi: $10.1155 / 2015 / 403012$.

11. Jia, B.B., Yang, Z.X., Mao, G.X., Lyu, Y.D, Wen, X.L., Xu, W.H., Lyu, X.L., Cao, Y.B., Wang, G.F. Health Effect of Forest Bathing Trip on Elderly Patients with Chronic Obstructive Pulmonary Disease. Biomed. Environ. Sci. $2016 ; 29: 212-218$.

12. Ohtsuka, Y. Walking in a Forest Is Beneficial for Type 2 Diabetic Patients; Li, Q., Ed.; Forest Medicine; Nova: Hauppauge, NY, USA, 2012; pp. 109-114.

13. Ohtsuka, Y. Effect of the Forest Environment on Blood Glucose; Li, Q., Ed.; Forest Medicine; Nova: Hauppauge, NY, USA, 2012; pp. 109-114.

14. Constantin Munteanu, Gabriela Dogaru. Bioclimatologie: Bioclimatologie umană. București: Editura Balneară,2020.

15. Gabriela Dogaru, Constantin Munteanu. Mofete: Gaze terapeutice. Bucureşti: Editura Balneară, 2020.

16. Munteanu Constantin, Munteanu Diana, Hoteteu Mihail, Dogaru Gabriela. Balneotherapy - Medical, Scientific, Educational And Economic Relevance Reflected By More Than 250 Articles Published In Balneo Research Journal. Balneo Research Journal. 2019;10(3):174-203.

17. Gabriela Dogaru, Alexandra Ispas, Ioana Stănescu, Marieta Motricala, Molnár Ákos. A clinical study on the efficacy of natural therapeutic factors in Băile Tuşnad for the rehabilitation of post-stroke patients. Balneo Research Journal. 2017;8(1):5-10.

18. Gabriela Dogaru, Ioana Stănescu, Daniela Pop, Marieta Motricală, Molnár Ákos. Effects of carbonated mineral water treatment in Băile Tuşnad on chronic arterial occlusive disease a case report. Balneo Research Journal. 2017;8(3):121-124. 